\title{
Motion of particles in a monolayer induced by coalescing of a bubble with a planar air-water interface
}

\author{
Xiangyang Ling a, b , Alexander Mayer ${ }^{\text {a }}$, Xingshi Yang ${ }^{a}$, Ghislain Bournival ${ }^{a}$ and Seher Ata ${ }^{\text {a* }}$ \\ ${ }^{a}$ School of Minerals and Energy Resources Engineering, University of New South Wales, Sydney, \\ NSW, 2052, Australia \\ ${ }^{\mathrm{b}}$ Current address: College of Chemistry and Chemical Engineering, Henan Polytechnic University, \\ Jiaozuo, China \\ *Corresponding author: s.ata@unsw.edu.au
}

\section{S1. Particle size characterization}

Soda-lime glass particles were supplied by Potters Industries Pty Ltd (Melbourne, Australia). The particles were divided into various size fractions by Ata ${ }^{1}$.The particles were mostly spherical in shape had a density of $2.5 \mathrm{~g} / \mathrm{cm}^{3}$. Most of the experiments were conducted using particles with a characteristic size of $d_{90}=96 \mu \mathrm{m}$ (the size at which $90 \%$ of particles are smaller or equal to that size) and a Sauter diameter of $d_{32}=65 \mu \mathrm{m}$ (volume/surface equivalent diameter). Smaller particles, distribution 2, and coarser particles, distribution 3 , were used to generate binary mixtures. The $d_{90}$ and $d_{32}$ of the particles for the different sizes and the particle size distributions for the particles are presented in Table S1 and Figure S1, respectively.

Table S1. The particle size of three groups used in the study. $d_{90}$ indicates the particle size of the fraction passing of $90 \%$ and $d_{32}$ is the Sauter mean diameter.

\begin{tabular}{ccc}
\hline Distribution & $d_{90}$ & $d_{32}$ \\
& $\mu \mathrm{m}$ & $\mu \mathrm{m}$ \\
\hline Distribution 1 & 96 & 65 \\
Distribution 2 & 64 & 44 \\
Distribution 3 & 134 & 93 \\
\hline
\end{tabular}




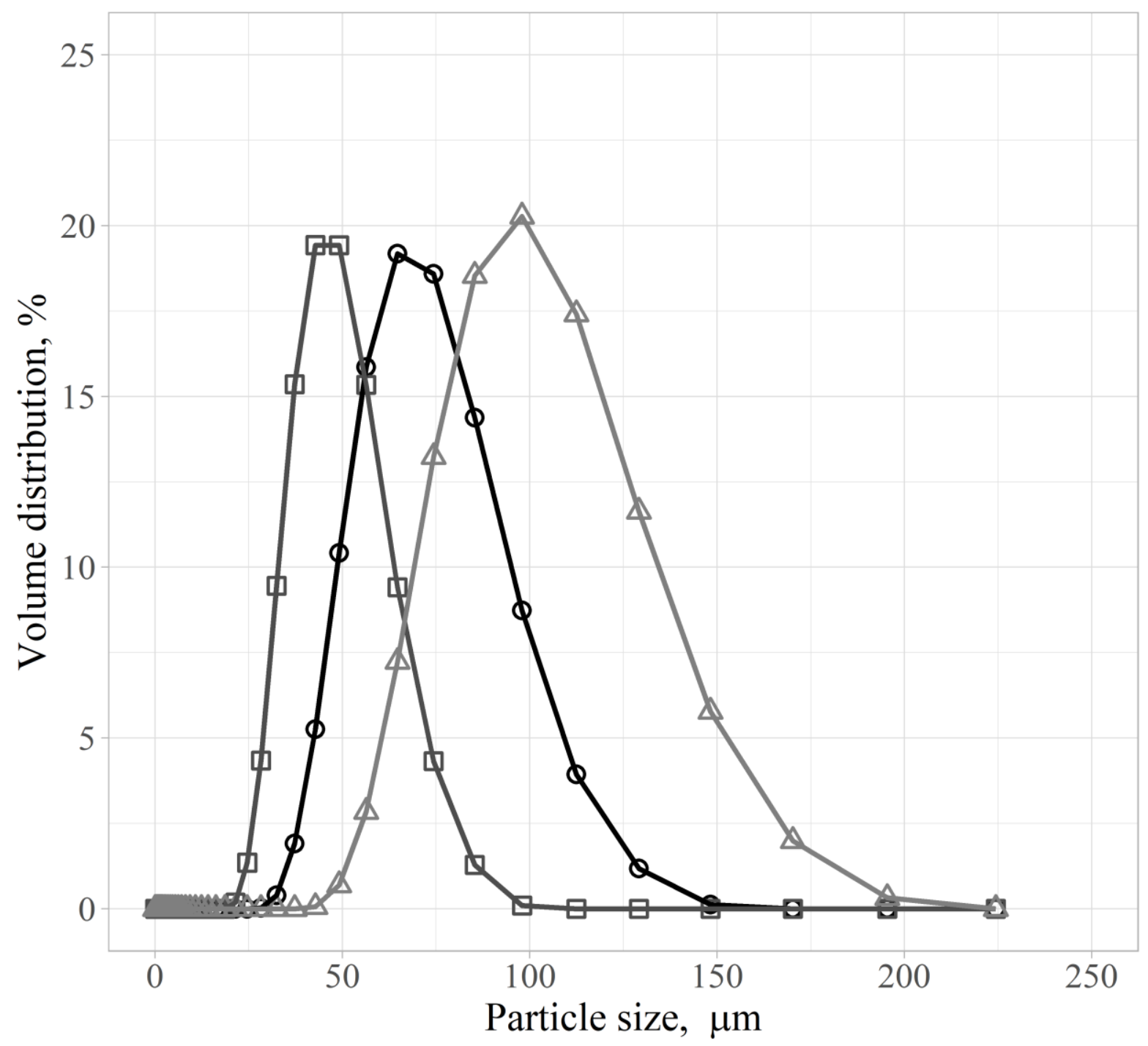

Figure S1. Size distribution of the particles used for experiments with (black circle - D1) unimodal distribution and the (dark grey square - D2) finer and (light grey triangle - D3) coarser particles used to generate bimodal distributions.

To achieve the bimodal monolayer, particles were mixed in known proportion on a weight basis using particles of two different size distributions (distribution 2 and distribution 3). In total five samples were prepared. The mixtures were characterized by the proportion of the coarser particles (distribution 3) in the sample on a weight basis $(\pi)$, and the polydispersity of the sample $(\eta)$, was calculated as: ${ }^{2}$

$$
\eta=\frac{\sqrt{\sum\left(d_{i}-d_{10}\right)^{2} \times n_{i} / \sum n_{i}}}{d_{10}}
$$

where, $d_{i}$ is the particle diameter in a given size fraction, $d_{10}$ is the arithmetic mean particle diameter (from the number distribution), $n_{i}$ is the number density with diameter $d_{i}$. The polydispersity number assumed a normal distribution and represents, in fact, the coefficient of variation (i.e. relative standard error). The calculated polydispersity values are shown in Table S2 whilst the resulting volume and number distributions are in Figure 2. 
Table S2. Characteristics of the particle mixtures showing the arithmetic (number) mean particle size, the standard deviation, and the relative standard error, which is used as a measure of polydispersity.

\begin{tabular}{cccccc}
\hline Mixture & 1 & 2 & 3 & 4 & 5 \\
\hline$\pi, \mathrm{wt} \%$ & 0 & 50 & 67 & 80 & 100 \\
$d_{N, 10}, \mu \mathrm{m}$ & 38.6 & 42.7 & 46.2 & 51.4 & 81.3 \\
$\sigma_{N, d 10}, \mu \mathrm{m}$ & 10.2 & 17.3 & 20.9 & 24.4 & 21.3 \\
$\eta$ & 0.26 & 0.40 & 0.45 & 0.47 & 0.26 \\
\hline
\end{tabular}
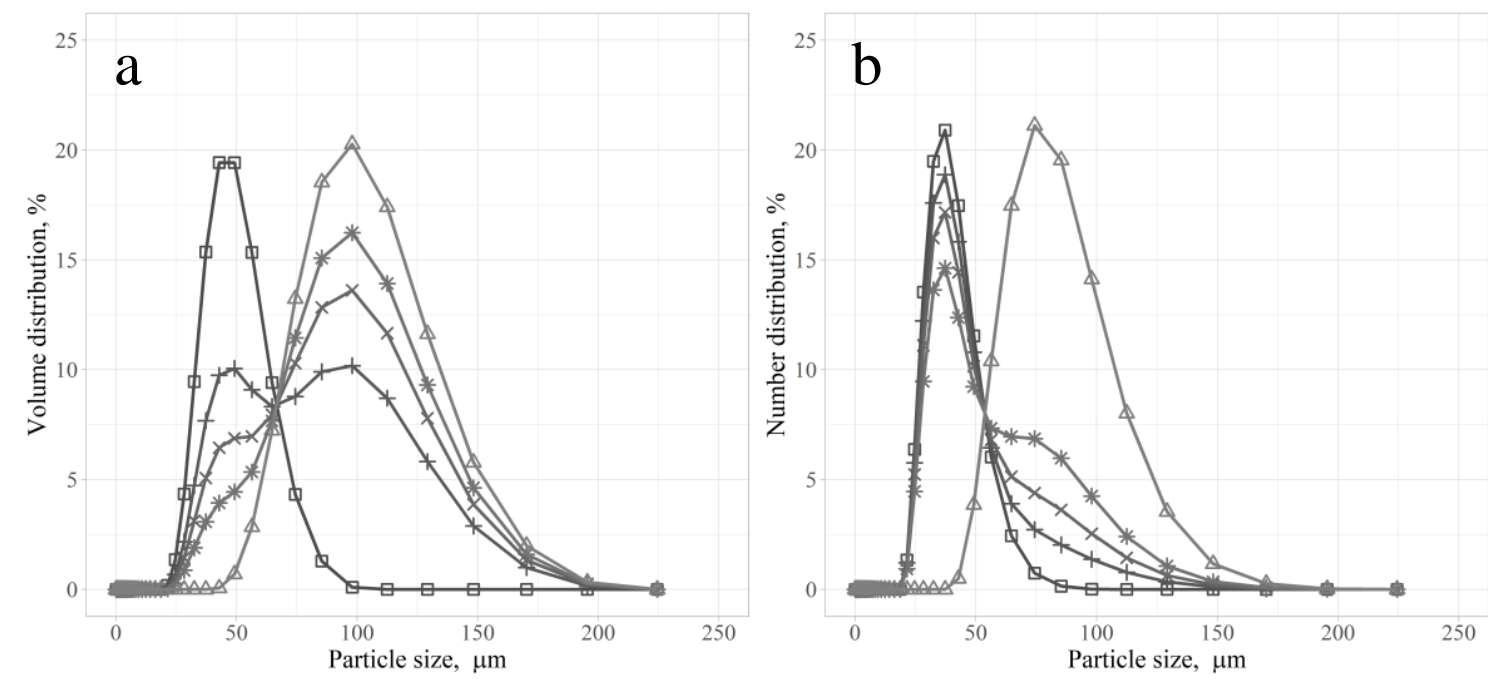

Figure 2. Size distribution of the mixtures of particles for (square) mixture 1 ( $0 \mathrm{wt} \%$ coarse particles, distribution 2 only), (plus) mixture 2 (50 wt $\%$ coarse particles), (cross) mixture 3 (67 wt $\%$ coarse particles), (star) mixture 4 (80 wt $\%$ coarse particles), and (triangle) mixture 5 (100 wt $\%$ coarse particles, distribution 3 only) for (a) the volume distribution and (b) the number distribution.

\section{S2. Particle contact angle}

The surface of the particles was esterified using 1-octanol (Sigma-Aldrich, $99 \%$ ) and 1-butanol (ChemSupply, $99 \%$ ). The hydrophobicity of the particles was determined from contact angle measurements on esterified glass plates. The contact angle was measured by submerging the plate in the modified Langmuir trough, which was filled with water topped with a layer of toluene, reproducing the experimental conditions for the production of a particle monolayer. Series of measurements were taken at $\mathrm{pH} 5.8$ and the $\mathrm{pH}$ was subsequently altered following different sequences using different plates. From a pH 5.8, the $\mathrm{pH}$ was lowered to a minimum of 3 and afterwards increased to a maximum of 9 . Alternatively, from $\mathrm{pH} 5.8$, the $\mathrm{pH}$ was increased to a maximum of 9 and then lowered to a minimum of 3. These different sequences were used to determine the effect of $\mathrm{pH}$ on the contact angle and to establish whether the $\mathrm{pH}$ altered the hydrophobic coating of the particles.

The mean contact angle measured at different $\mathrm{pH}$ are shown in Figure S3. It can be seen that the octanoltreated particles yielded higher contact angles than the butanol-treated particles as expected by the length of the hydrocarbon chain. ${ }^{3}$ While the values of the contact angle of the butanol-treated are 
comparable to that previously measured, the octanol-treated glass plate lower range of contact angles for this type of coating. ${ }^{4-5}$ However the plates were here wetted by toluene first as previously mentioned. A t-test was performed to compare the effect of the sequence (i.e. acid to alkaline or alkaline to acid) on the contact angle with a significance level of 0.05 . The p-values are shown as an insert in Figure S3 for each $\mathrm{pH}$. It can be seen that the sequence under which the contact angles were measured had a negligible effect on the contact angle suggesting that the coating was remained intact under acidic or alkaline conditions. Although at p-value less than the significance level is generally used as a rejection criterion, it should be noted that the p-value is the probability that the difference in mean value between the two measurements will take on a value at least as extreme as the estimated values when the null hypothesis (i.e. $\mathrm{H}_{0}: \beta_{a c i d, p H i}-\beta_{\text {alkaline, } p H i}=0$ ) is true ${ }^{6}$ In all cases, such a probability is high. As such, the results for each $\mathrm{pH}$ have been combined.

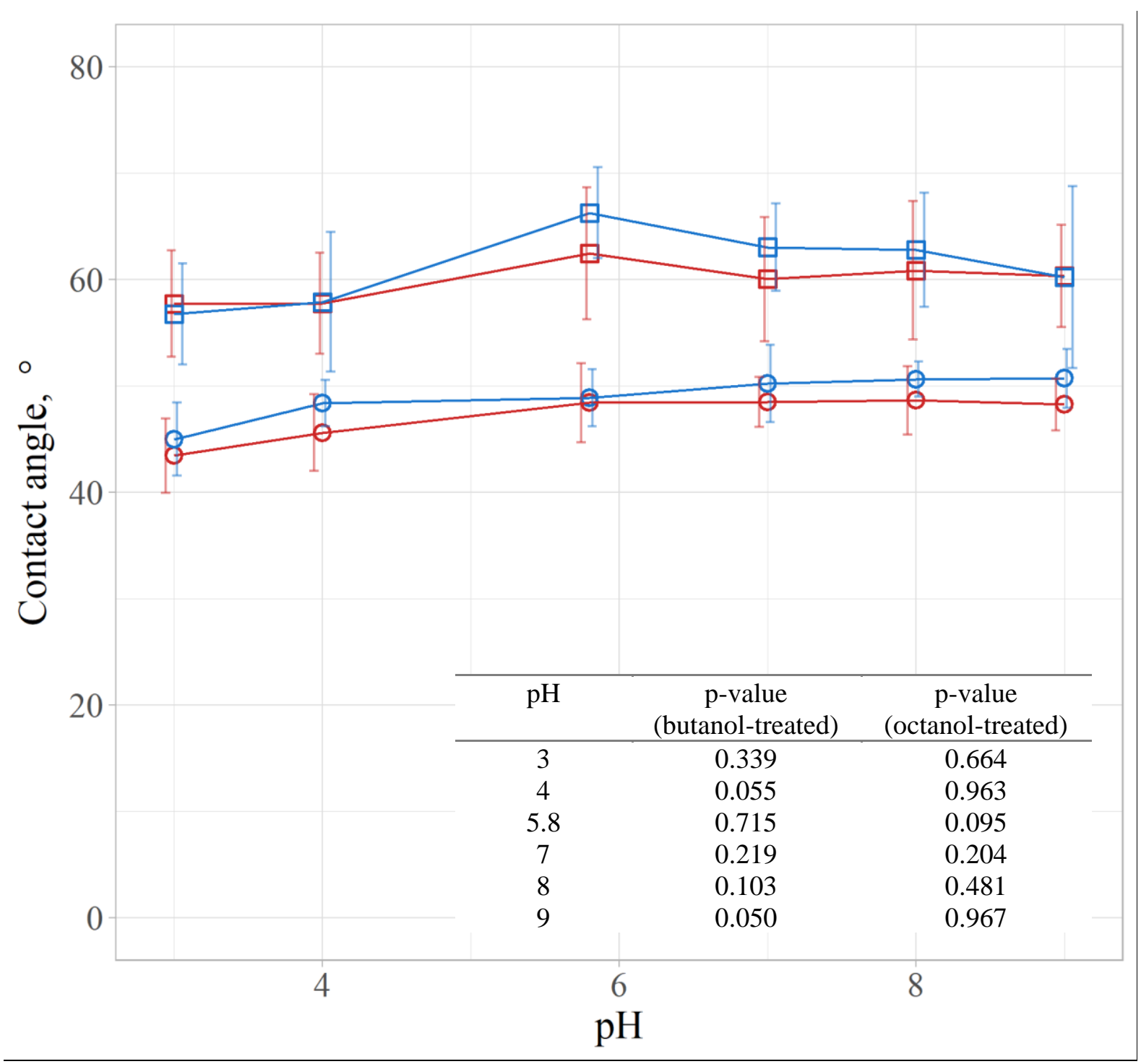

Figure S3. Effect of the $\mathrm{pH}$ on the mean contact angle of hydrophobized glass plates. The circles represent the butanol-treated glass plates and the squares represent the octanol-treated glass plates. The series in blue shows the effect of increasing the $\mathrm{pH}$ and then lowering the $\mathrm{pH}$ whilst the series in 
red shows the effect of lowering the $\mathrm{pH}$ and then increasing the $\mathrm{pH}$. The error bars represent one standard deviation from at least 10 measurements. The $\mathrm{p}$-values from a two-sided t-test comparing the effect of the sequence in the change of $\mathrm{pH}$ are also included for a significance level $(\alpha)$ of 0.05 .

To compare more than two groups, a one-way analysis of variance (ANOVA) test is used. ${ }^{7}$ The p-value of the ANOVA test is in this case the probability that in any of the $\mathrm{pH}$ subgroups, the difference in the mean contact angle will take on a value at least as extreme as the estimated values when the null hypothesis (e.g. $\mathrm{H}_{0}: \mu_{\text {butanol } p H i}-\mu_{\text {butanol, } p H j}=0$ ) is true. This probability was $1.55 \times 10^{-8}$ for the butanoltreated particles and $1.47 \times 10^{-8}$ for the octanol-treated particles. Thus, $\mathrm{pH}$ is shown to influence the contact angle of the particles.

A Tukey HSD (Honest Significant Difference) test was conducted as a post hoc test on the datasets to compare all the pairwise combinations and build a confidence interval on the difference in the mean values for each pair. ${ }^{6}$ The Tukey HSD difference of means plot for butanol- and octanol-treated particles are shown in Figure 4 for a 95\% confidence interval. The plots confirm that there exist differences in contact angles depending on the $\mathrm{pH}$. In the case of the butanol-treated particles, the contact angle measured at $\mathrm{pH} 3$ is significantly different from all other measured contact angles except at $\mathrm{pH} 4$. For octanol-treated particles, the contact angle in which acid was added showed a significant difference from that measured at $\mathrm{pH} 5.8$.
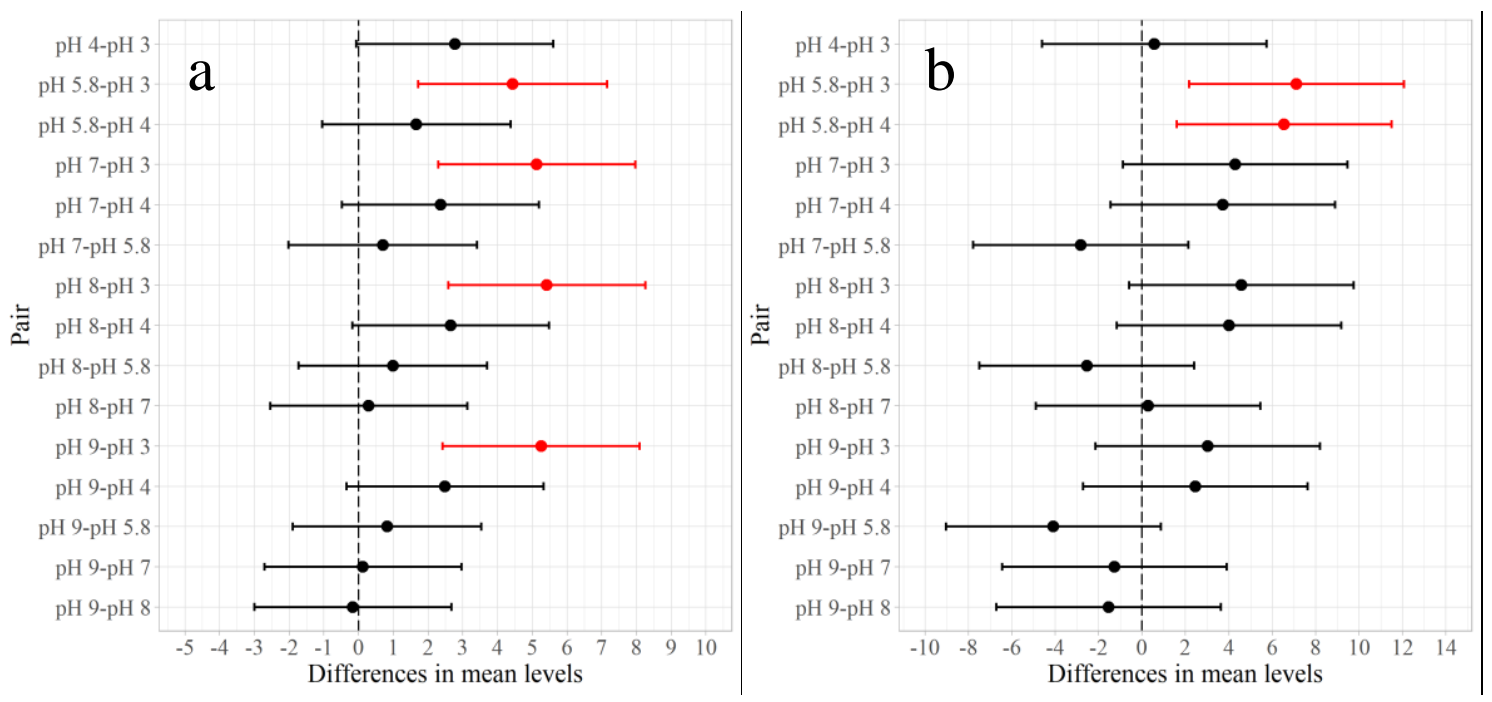

Figure 4. Graphical display of pairwise comparison of differences in mean contact angle between different $\mathrm{pH}$ from Tukey's HSD test with a 0.05 confidence level. The comparison shown for (a) the butanol-treated particles and for (b) the octanol-treated particles. A bar in red represents a significantly different difference in mean values.

In some instances, the experiments were conducted at different $\mathrm{pH}$. The butanol-treated particles were

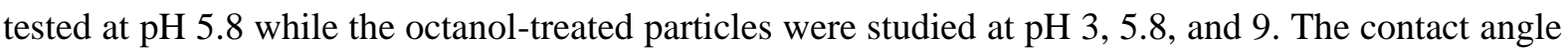
for butanol-treated particles at $\mathrm{pH} 5.8$ was taken as the mean value of all measurements except those at $\mathrm{pH}$ 3. In the case of octanol-treated particles, the contact angle at the given $\mathrm{pH}$ was taken. The relevant 
contact angles are presented in Table S3. It should be noted that Laskowski and Kitchener ${ }^{8}$ observed a variation in the contact angle of methylated silica at various $\mathrm{pH}$.

Table S3. Contact angle measured at various $\mathrm{pH}$ on glass plates esterified in different alcohols.

\begin{tabular}{ccccc}
\hline Treatment & $\mathrm{pH}$ & $\begin{array}{c}\text { Contact angle } \\
\left({ }^{\circ}\right)\end{array}$ & $\begin{array}{c}\text { Standard deviation } \\
\left({ }^{\circ}\right)\end{array}$ & $\begin{array}{c}\text { Number of } \\
\text { measurements }\end{array}$ \\
\hline Butanol & 5.8 & 48.8 & 3.1 & 104 \\
Octanol & 3 & 57.3 & 4.8 & 20 \\
Octanol & 5.8 & 64.4 & 5.6 & 24 \\
Octanol & 9 & 60.3 & 6.7 & 20
\end{tabular}

\section{S3. Surface tension}

\section{S3.1 Dynamic surface tension of PPG}

Once frother molecules adsorb at the air-water interface from the solution, the molecules may, like in the case of PPG 425, undergo rearrangement at the interface. ${ }^{9}$ The surface tension drops quickly at the beginning due to a diffusion-controlled process followed by a slow molecular reorganization process. A relaxation process for PPG 425 was pointed out by Bournival, et al. ${ }^{10}$ but is not significant at lower concentrations. The surface tension of PPG 425 solutions was measured at concentrations ranging from $10^{-6} \mathrm{M}$ to $10^{-4} \mathrm{M}$ using a PAT-1M (Sinterface Technology, Germany). The results are plotted as a function of time in Figure S5. It is evident that the surface tension consistently decreased over a long time period at higher concentrations. There is indication of relaxation for concentrations of at least $10^{-}$ ${ }^{4} \mathrm{M}$. 


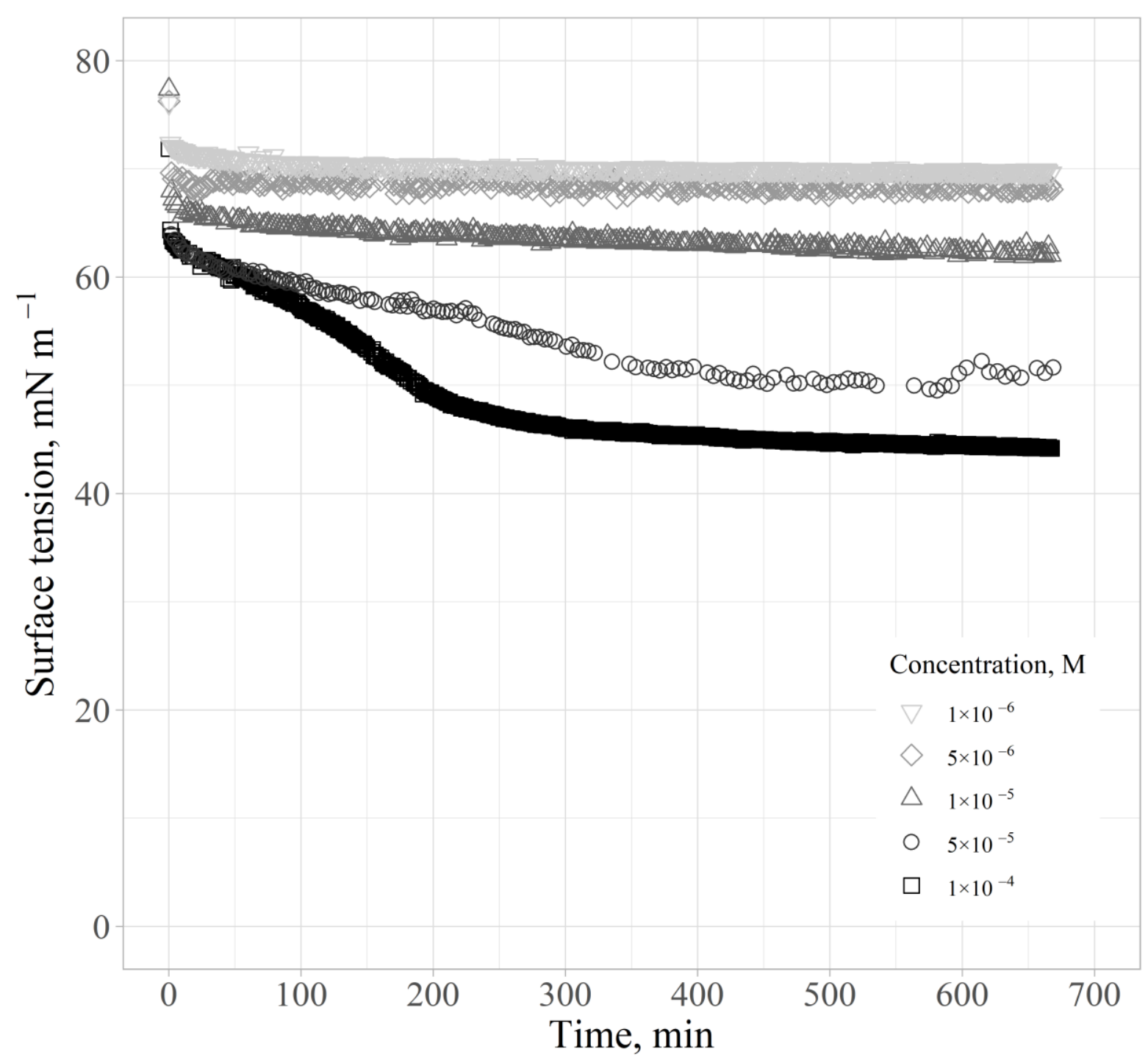

Figure S5. Dynamic surface tension of PPG 425 solutions at various concentrations.

\section{S3.2 Surface tension of concentrations tested}

The frother concentrations in this study were determined based on the variation in surface tension and the relaxation process. For 1-pentanol and MIBC, the concentrations studied covered both low concentrations where the surface tension is relatively constant and the concentration range where the surface tension tends to decrease (as observed on the log scale of the equilibrium surface tension as a function of the added concentration). For PPG 425, the time-scale of the experiment was kept shorter than the time required to reach an equilibrium. The aging time for this frother was set at $15 \mathrm{~min}(900 \mathrm{~s})$. The frothers and concentrations used in the study are listed in Table S4. The value obtained is relatively comparable with literatures. ${ }^{10-11}$

Table S4. List of the frother concentrations selected in the study and the measured surface tension of the solutions with a $99 \%$ confidence interval.

$\begin{array}{lll}\text { Reagent } & \text { Concentration } & \text { Surface tension }\end{array}$




\begin{tabular}{ccc}
\hline & $\mathrm{M}$ & $\mathrm{mN} / \mathrm{m}$ \\
\hline 1 1-pentanol & $1 \times 10^{-4}$ & $71.5 \pm 1.0$ \\
& $1 \times 10^{-3}$ & $71.0 \pm 0.3$ \\
& $1 \times 10^{-2}$ & $65.5 \pm 1.2$ \\
& $1 \times 10^{-1}$ & $45.0 \pm 3.9$ \\
MIBC & $1 \times 10^{-5}$ & $72.4 \pm 0.4$ \\
& $1 \times 10^{-4}$ & $72.0 \pm 0.5$ \\
& $1 \times 10^{-3}$ & $70.2 \pm 0.5$ \\
& $1 \times 10^{-6}$ & $70.1 \pm 0.1$ \\
PPG 425 & $5 \times 10^{-6}$ & $68.5 \pm 0.6$ \\
& $1 \times 10^{-5}$ & $63.5 \pm 0.1$ \\
& $5 \times 10^{-5}$ & $51.4 \pm 0.3$ \\
& $1 \times 10^{-4}$ & $44.1 \pm 0.1$
\end{tabular}

\section{S4. Interaction forces between two particles}

\section{S4.1 Effect of particle hydrophobicity}

The forces acting on a particle at a gas-liquid interface have a significant influence on the particle motion and have been calculated in relation to froth flotation extensively. ${ }^{12-16}$ Major forces acting on a particle attached to a stationary free surface at equilibrium are buoyancy, capillary force, hydrostatic force and particle weight. ${ }^{17}$ Of these, the first three are adhesive forces and show the strength of the particle attachment while particle weight is considered the disruptive force. If the interface is disturbed by some external energy inputs, such as ultrasonic or bubble coalescence, additional forces such as the drag force, inertial force and Basset history force should also be considered in the force balance. ${ }^{15}$ In particular, it should be noted that the Basset history force is often insignificant compared to drag and inertial forces in fluid-particle systems due to the relatively low angular frequency. Thus, the Basset history force is ignored in this study.

The main forces acting on a particle attached to an air-water interface are as follows:

1. The buoyancy is given by the product of the density of the liquid, acceleration due to gravity, and the volume of liquid displaced by the submerged section of the particle, that is:

$$
F_{b}=\rho_{f} g \frac{\pi r_{p}^{3}}{3}\left(2+3 \cos \alpha-\cos ^{3} \alpha\right)
$$

where is the radius of the particle, $\rho_{f}$ is the liquid density, $\alpha$ is the half angle subtended by the three-phase contact line at the particle center.

2. The capillary force acts along the tangent to the gas-liquid interface at the three-phase contact line and tends to pull the particle into the gas phase. The surface tension can be resolved into the vertical and horizontal forces. Due to the rotational symmetry along the vertical axis, the 
horizontal component of the capillary force vanishes and only the vertical component remains. ${ }^{18}$ The vertical component of the capillary force is:

$$
F_{c}=2 \pi \sigma r_{p} \sin \alpha \sin (\theta-\alpha)
$$

3. The hydrostatic force also stabilizes the particle attachment to the gas-liquid interface, which comes from the hydrostatic pressure over the area enclosed by the three-phase contact line. ${ }^{18}$ This force can be described by:

$$
F_{h}=\pi r_{p}^{2} \rho_{f} g L H \sin \alpha
$$

where $H$ is the height of the gas-liquid meniscus over the three-phase contact, $H=r_{t p c} \sin \left(\frac{\theta}{2}\right)\left(\ln \frac{4 L / r_{t p c}}{1+\cos \left(\frac{\theta}{2}\right)}-0.577\right), r_{t p c}$ is the radius of the three-phase contact, $r_{t p c}=$ $r_{p} \sin \left(\frac{\theta}{2}\right), L$ is the capillary length given by: $L=\sqrt{\frac{\sigma}{\rho_{f} g}}$.

4. The particle weight is:

$$
F_{w}=-\frac{4 \pi r_{p}^{3}}{3} \rho_{p} g
$$

where $\rho_{p}$ is the particle density, $r_{p}$ is the radius of a spherical particle, $g$ is the acceleration due to gravity.

5. The drag force can be estimated by integrating the shear and normal stresses predicted by Stokes analysis for the drag on an isolated sphere over the wetted surface of the particle at low Reynolds number, ${ }^{15}$ that is:

$$
F_{d}=-\pi \mu u_{\theta} r_{p}\left(1+\cos ^{3} \alpha+4(2-\cos \alpha) \cos ^{4} \frac{\alpha}{2}\right)
$$

where $\mu$ is the viscosity and $u_{\theta}$ is the moving velocity of a sphere particle at the interface.

6. The inertial force is given by the effective mass (i.e., the particle mass plus added mass) multiplied by the acceleration of the particle, and acts in the opposite direction to the acceleration. Since the added mass is given by one-half of the displaced mass of liquid, the inertial force is estimated to be: ${ }^{15}$

$$
F_{i}=-\frac{\pi r_{p}^{3}}{6} a_{\theta}\left(8 \rho_{p}+\rho_{f}\left(2+3 \cos \alpha-\cos ^{3} \alpha\right)\right)
$$

where $a_{\theta}$ is the acceleration of a sphere particle at the interface.

Taking the density as $2.5 \mathrm{~g} / \mathrm{cm}^{3}$, the average weight was estimated to be $-0.0087 \mu \mathrm{N}$. The adhesive forces given by Equations 2-4 are all functions of the position of the three-phase contact and reach a maximum when the central angle is half of the contact angle. ${ }^{18}$ The dynamic viscosity of the water at $25{ }^{\circ} \mathrm{C}$ is $0.8891 \times 10^{-3} \mathrm{~Pa} \cdot \mathrm{s}$, thus for the less and more hydrophobic particles, the corresponding buoyancy, capillary force and hydrostatic force are $0.0035 \mu \mathrm{N}, 3.43 \mu \mathrm{N}$ and $0.0011 \mu \mathrm{N}$, while for the more hydrophobic particles these forces are $0.0034 \mu \mathrm{N}, 5.72 \mu \mathrm{N}$ and $0.0003 \mu \mathrm{N}$, respectively. Since the 
magnitude of the particle weight, buoyancy and hydrostatic force are relatively small compared to the capillary force, the particles were less likely to detach from the interface. After the bubbles coalesce with the flat interface, the particle movement and rearrangement at the air-water interface was not only influenced by the lateral interparticle forces which included the van der Waals force, the electrostatic force and the capillary force, but also by the lateral fluid hydrodynamics which included the drag force and inertial force. The magnitude of drag force is proportional to the particle speed (as shown in Eq 6), while the inertial force is proportional to the particle acceleration (Eq 7). It is important to note that both the particle speed and acceleration as a function of time can be obtained through the particle tracking methodology used in this study. Therefore, the lateral drag and inertial forces can also be calculated under the assumption that the central angle was half the contact angle.

Figure S6 shows the magnitude of lateral drag and inertial forces with respect to time for the less and more hydrophobic particles. The calculated lateral drag force and inertial force were less than $10^{-5} \mathrm{~N}$ in magnitude. The calculation of the interaction force between two identical particles ${ }^{19}$ has been updated for the measured contact angles and are presented in Figure S7 (see insert). A mininum in the interaction forces was calculated to be $-0.00118 \mu \mathrm{N}$ and $-0.00216 \mu \mathrm{N}$, for less hydrophobic and more hydrophobic particles, respectively. The drag and inertial forces are some order of magnitude higher than the interaction forces (under the assumptions made in the calcalculations) indicating that the raft could break if the monolayer in the vicinity of the bubble was not continuous. In fact, Poulichet and Garbin ${ }^{20}$ also found that the magnitude of the drag force tangential to the interface is comparable to the attractive interparticle interactions and can therefore cause the breakup of colloidal particle aggregates, until the particles are uniformly dispersed at the interface.

The detachment force experienced by the particles was influenced by the hydrophobicity of the particles as indicated in Figure S6. That is, the less hydrophobic particles experienced both a larger drag force and inertial force than the more hydrophobic particles. The difference in force exerted on the particles is suggesting that the less hydrophobic particles were more easily re-arranged on the surface. It is also expected that due to an external force the less hydrophobic particles, which have a lower adhesion force and a greater detachment force, will be more likely to detach as reported by others. ${ }^{1,14,21-22}$ 

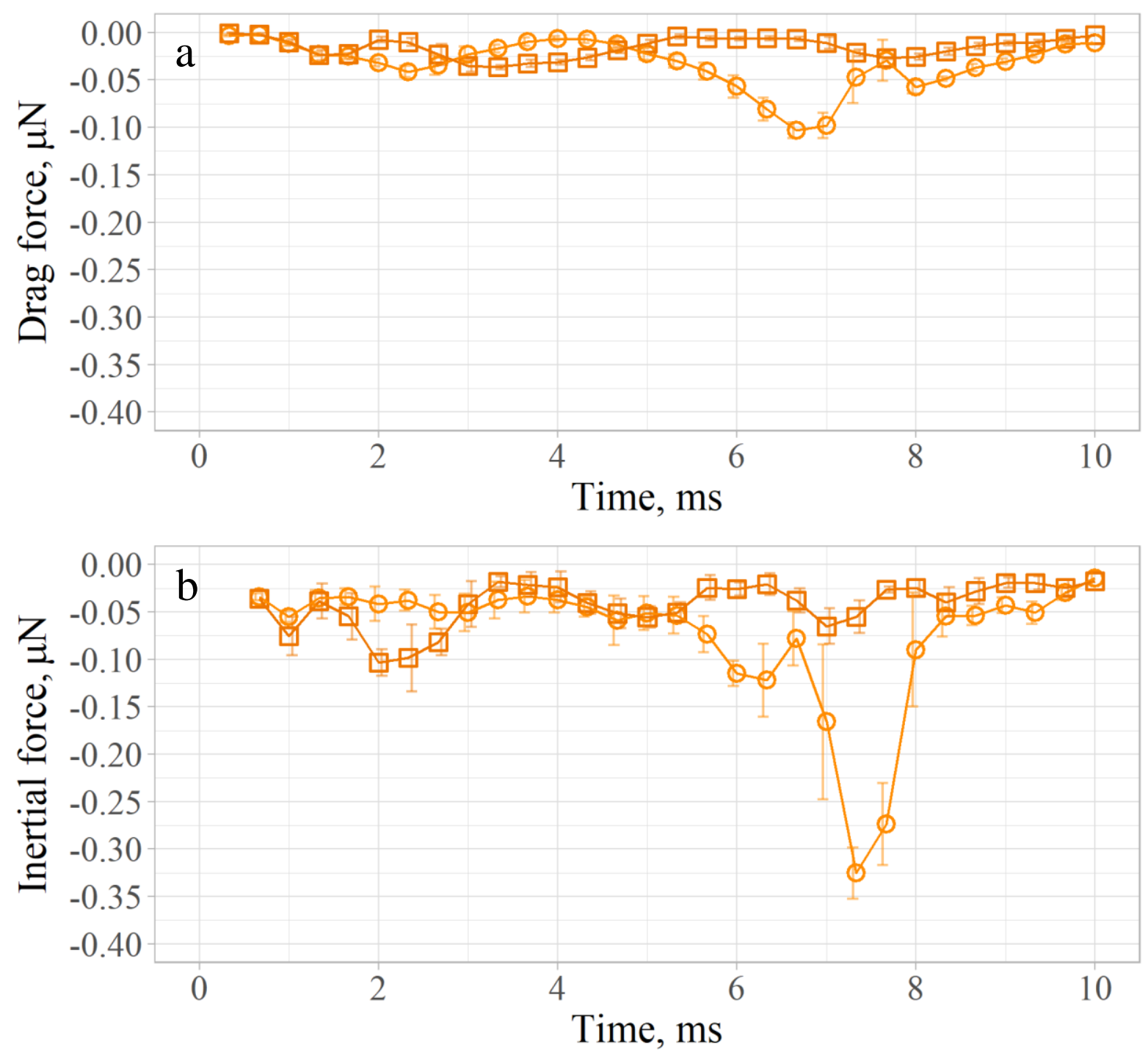

Figure S6. The (a) lateral drag and (b) inertial forces as a function of time for particles (circle) butanol-treated particles $\left(d_{32 \text {, particle }}=65 \mu \mathrm{m}\right.$, contact angle $\left.=48.8^{\circ}\right)$ and (square) octanol-treated particles (contact angle $\left.=64.4^{\circ}\right)(\mathrm{pH}=5.8$, bubble size $=2.1 \pm 0.05 \mathrm{~mm}$, packing factor $=76 \pm 2 \%$ ). 


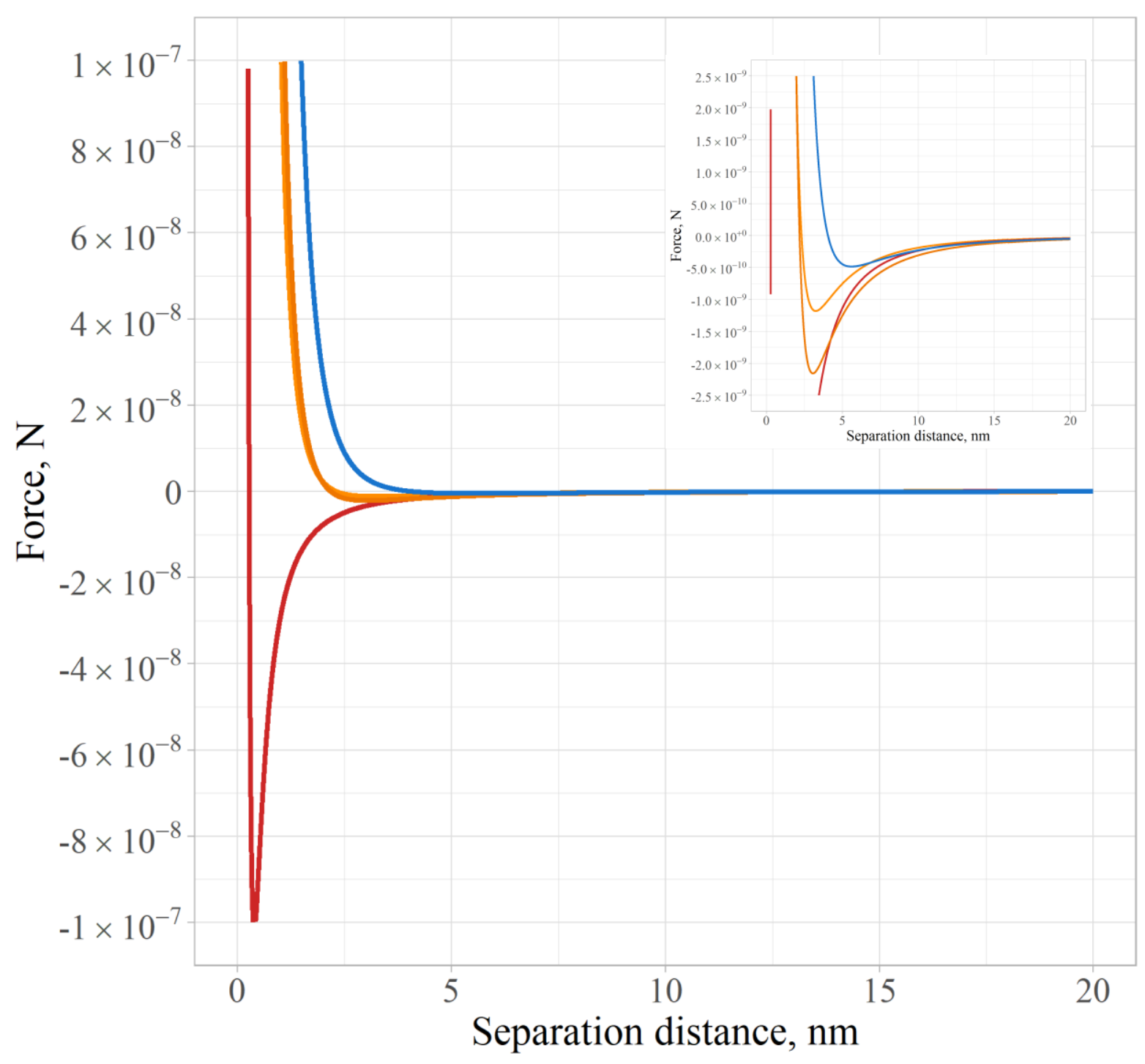

Figure S7. The interaction force between two identical silica particles of size $65 \mu \mathrm{m}$ as a function of the separation distance at $\mathrm{pH}$ (red) 3, (orange) 5.8, and (blue) 9 for the octanol-treated particles. The

butanol-treated particles at $\mathrm{pH} 5.8$ is also in orange. The inset highlights the difference in the interaction force between the less and more hydrophobic particles (both at $\mathrm{pH}$ 5.8). The calculations were performed using the assumptions detailed in Bournival, et al. ${ }^{14}$ with the measured contact angles from Section 2.1 of the main article. The zeta potentials were assumed to be $-5 \mathrm{mV},-40 \mathrm{mV}$, and -70 $\mathrm{mV}$ at $\mathrm{pH} 3,5.8$, and 9, respectively as approximated from Bergna and Roberts ${ }^{23}$.

\section{S4.2 Effect of subphase pH}

The forces (Equations 2 - 7) were similar to that presented in section S4.1 for the octanol-treated particles. The values were affected as much as the values of the contact angles were affected. The $\mathrm{pH}$ of the subphase is known to influence the surface force.

Clean silica surfaces hydrate due to short-range hydration forces and their surface charge is strongly dependent on $\mathrm{pH}$ and ionic strength of the subphase. ${ }^{24-25} \mathrm{At} \mathrm{pH}$ above the isoelectric point (IEP = 2 - 3), the silica surfaces are negatively charged and the charge density increases as the $\mathrm{pH}$ increases. ${ }^{26}$ The 
zeta potential of glass beads at $\mathrm{pH} 3,5.8$, and 9 is approximately $-5 \mathrm{mV},-40 \mathrm{mV}$ and, $-70 \mathrm{mV}$, respectively. ${ }^{23}$ Thus, the repulsive force between the particles is expected to become increasingly important as the $\mathrm{pH}$ increases from 3 to 9 due to increases of the negative charge density on the particles as reported in Figure S7. Generally, the larger the repulsive force between the particles, the weaker the structural strength of the particle monolayer and the stronger the particle motion at the interface. This trend was observed for the compression of the monolayer and the calculation of the pressure inherent to the monolayer. ${ }^{19}$ However, when subjected to a rapid change, the mobility of the particles at $\mathrm{pH} 3$ was greater than theoretically anticipated. A possible degradation of the coating at $\mathrm{pH} 3$ was not considered important as mentioned in Section 2.1 of the main article.

Despite the change in the contact angle, the minimum in the force curve is strongly influenced by the charge of the particles, as previously pointed out. However, the minimum in the curve occurs at a distance of less than a couple of nanometers. The length of an octane molecule is approximately 3.33 $\AA^{27}$, which may prevent the particle from optimal electrostatic interaction but may give rise to surface interactions (i.e. similar to polymer brushes). Albeit, the $\mathrm{pH}$ of the subphase did influence to some extent the interaction of the particles as observed in the formation of the monolayers. ${ }^{19,28}$ It is known that similar to simple alcohols, the hydrocarbon chain will form hydrogen bonds. ${ }^{29}$ However the effect of $\mathrm{pH}$ on this interaction is still unknown and cold have affected the particle mobility.

\section{S5. Effect of polydispersity on the motion of particles}

The effect of particle polydispersity on the motion of particles was tested. Measurements were conducted on finer and coarser particles to determine if they behaved differently in the monolayer. Figure S8 shows the maximum in the MSD as a function of particle size and polydispersity index. The maximum MSD of finer and coarser particles in a more loosely packed monolayer appears to be higher for a low polydispersity index (which was obtained from fine only and coarse only particle size distribution). Otherwise, no noticeable trend could be discerned with the particle size distributions employed. 

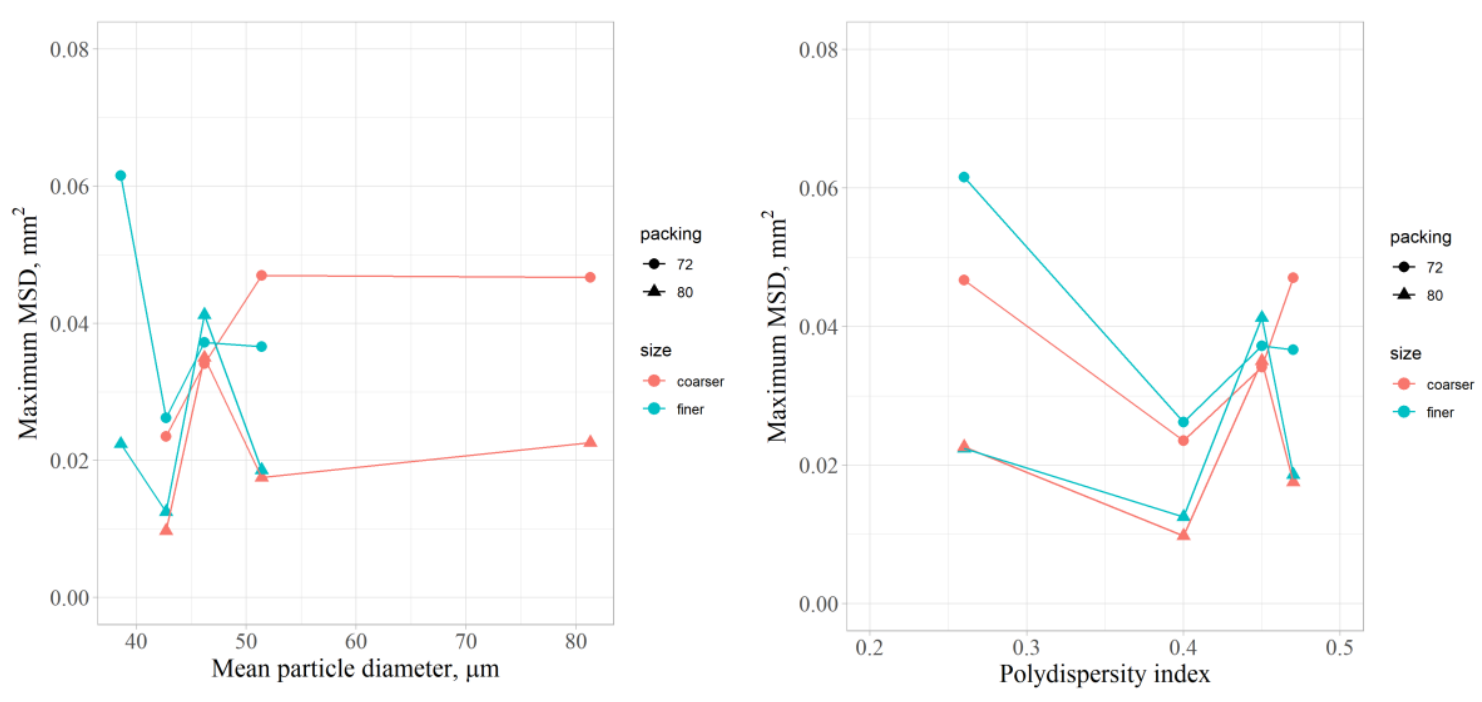

Figure S8. Maximum MSD for coarser and finer particles in layers less densely packed and more densely packed monolayers expressed as a function of (left) mean particle size and (right) polydispersity index.

\section{References}

1. Ata, S., The detachment of particles from coalescing bubble pairs. Journal of Colloid and Interface Science 2009, 338 (2), 558-565.

2. Chu, X.; Nikolov, A. D.; Wasan, D. T., Effects of particle size and polydispersity on the depletion and structural forces in colloidal dispersion. Langmuir 1996, 12 (21), 5004-5010.

3. Kimura, T.; Kuroda, K.; Sugahara, Y.; Kuroda, K., Esterification of the silanol groups in the mesoporous silica derived from kanemite. Journal of Porous Materials 1998, 5, 127-132.

4. Bournival, G.; Ata, S.; Jameson, G. J., The influence of submicron particles and salt on the recovery of coarse particles. Minerals Engineering 2014, 69, 146-153.

5. Hunter, T. N.; Wanless, E. J.; Jameson, G. J., Effect of esterically bonded agents on the monolayer structure and foamability of nano-silica. Colloids and Surfaces A: Physicochemical and Engineering Aspects 2009, 334 (1-3), 181-190.

6. Montgomery, D. S.; Runger, G. C., Applied statistics and probability for engineers. John Wiley \& Sons, Inc.: New York, 2003; p 706.

7. Nayak, B. K.; Hazra, A., How to choose the right statistical test? Indian Journal of Ophthamology 2011, 59 (2), 85-86.

8. Laskowski, J.; Kitchener, J. A., The hydrophilic-hydrophobic transition on silica. Journal of Colloid and Interface Science 1969, 29 (4), 670-679.

9. Díez-Pascual, A. M.; Compostizo, A.; Crespo-Colín, A.; Rubio, R. G.; Miller, R., Adsorption of water-soluble polymers with surfactant character. Adsorption kinetics and equilibrium properties. Journal of Colloid and Interface Science 2007, 307 (2), 398-404.

10. Bournival, G.; Ata, S.; Karakashev, S. I.; Jameson, G. J., An investigation of bubble coalescence and post-rupture oscillation in non-ionic surfactant solutions using high-speed cinematography. Journal of Colloid and Interface Science 2014, 414, 50-58.

11. Bournival, G.; de Oliveira e Souza, L.; Ata, S.; Wanless, E. J., Effect of alcohol frothing agents on the coalescence of bubbles coated with hydrophobized silica particles. Chemical Engineering Science 2015, 131, 1-11.

12. Schulze, H. J., New theoretical and experimental investigations on stability of bubble/particle aggregates in flotation: A theory on the upper particle size of floatability. International Journal of Mineral Processing 1977, 4 (3), 241-259.

13. Singh, P.; Joseph, D. D., Fluid dynamics of floating particles. Journal of Fluid Mechanics 2005, $530,31-80$. 
14. Bournival, G.; Ata, S.; Wanless, E. J., The behavior of bubble interfaces stabilized by particles of different densities. Langmuir 2016, 32 (25), 6226-6238.

15. Stevenson, P.; Ata, S.; Evans, G. M., The behavior of an oscillating particle attached to a gasliquid surface. Industrial and Engineering Chemistry Research 2009, 48 (17), 8024-8029.

16. Nutt, C. W., Froth flotation: The adhesion of solid particles to flat interfaces and bubbles. Chemical Engineering Science 1960, 12 (2), 133-141.

17. Nguyen, A. V.; Schulze, H. J., Colloidal science of flotation. Marcel Dekker, Inc.: New York, 2004; Vol. 118, p 850.

18. Nguyen, A. V., New method and equations for determining attachment tenacity and particle size limit in flotation. International Journal of Mineral Processing 2003, 68 (1-4), 167-182.

19. Yang, X.; Mayer, A.; Bournival, G.; Pugh, R. J.; Ata, S., Experimental technique to study the interaction between a bubble and the particle-laden interface. Frontiers in Chemistry 2018, 6, 348.

20. Poulichet, V.; Garbin, V. In Ultrafast desorption of colloidal particles from fluid interfaces, Weitz, D. A., Ed. National Academy of Sciences: 2015; pp 5932-5937.

21. Tan, S.-Y.; Ata, S.; Wanless, E. J., Direct observation of individual particle armored bubble interaction, stability, and coalescence dynamics. Journal of Physical Chemistry B 2013, 117 (28), 85798588 .

22. Rahman, R. M.; Ata, S.; Jameson, G. J., Study of froth behaviour in a controlled plant environement - Part 2: Effect of collector and frother concentration. Minerals Engineering 2015, 81, 161-166.

23. Bergna, H. E.; Roberts, W. O., Colloidal silica: Fundamentals and applications. 1st ed.; Taylor \& Francis Group: New York, 2006; p 895.

24. Behrens, S. H.; Grier, D. G., The charge of glass and silica surfaces. The Journal of Chemical Physics 2001, 115 (14), 6716-6721.

25. Binks, B. P.; Duncumb, B.; Murakami, R., Effect of $\mathrm{pH}$ and salt concentration on the phase inversion of particle-stabilized foams. Langmuir 2007, 23 (18), 9143-9146.

26. Elimelech, M., Indirect evidence for hydration forces in the deposition of polystyrene latex colloids on glass surfaces. Journal of the Chemical Society, Faraday Transactions 1990, 86 (9), 16231624 .

27. Laskowski, L.; Burk, R. E., The probable length of hydrocarbon chains. Journal of Chemical Physics 1939, 7 (7), 465-469.

28. Máté, M.; Zrínyi, M.; Horvölgyi, Z., Structure formation and interaction of silanized glass beads at water-fluid interfaces: a redispersability study. Colloids and Surfaces A: Physicochemical and Engineering Aspects 1996, 108 (2-3), 147-157.

29. Gélinas, S.; Finch, J. A.; Gouet-Kaplan, M., Comparative real-time characterization of frother bubble thin films. Journal of Colloid and Interface Science 2005, 291 (1), 187-191. 